\title{
The Influence of Adsorption on Electrode Processes and on Some Substitution Chemical Reaction Studied by Polarography
}

\author{
By \\ Jaroslav KU゚TA \\ Polarographic Institute of the Czechoslovak Academy of Sciences, Prague \\ To the honour of Professor Isamu Tachi
}

(Received Nov. 18, 1962)

By means of polarography very important physico-chemical data, e.g., the equilibrium constants, constants of very fast chemical reactions, rate constants of some electrochemical reactions etc. can be easily obtained. From the shift of the half-wave potentials with substituents in analogous series of substances some interesting correlations were established.

The correctness of obtained data and constants may be influenced by some complicating factors. Among them the adsorption of the depolarizer or of the depolarization product or of some other electrochemically inactive substances present in the solution must be considered. Of course the complicating influence of the adsorption is not restricted to the use of the polarographic method. When using the other electrochemical methods developed in recent years ${ }^{1,2)}$ and based very often on the previous experience of polarography the influence of adsorption must be taken into account.

On the other hand from the influence of the adsorption on electrochemical kinetics some important adsorption parameters can easily be obtained. This, we can say, indirect method of the study of the adsorption is very often more sensitive than the direct methods for the investigation of adsorption, e.g. electrocapillary curves, differential capacity measurement etc.

E.g., the specific adsorption of $\mathrm{Cs}^{+}$was found in the first place from the accelerating effect $^{4,5)}$ exhibited on the reduction rates of reducible anions and then the direct proof $f^{5,6)}$ from electrocapillary curves was done.

In the following mainly the results concerning the influence of foreign electroinactive substances on the electrode processes occuring at the dropping mercury electrode obtained in our Institute will be mentioned. A more complete list of data and references can be found in reviews ${ }^{7,8)}$ which appeared recently.

It is very well-known to all polarographers that a small addition of surface active substances is sometimes necessary in order to eliminate the maxima of the I. and II. kind. However, at certain concentrations of these substances, even in traces a strong and often insidious effect on electrode processes can be observed.

Thus, the surface active substances have a great influence on the polarographic waves, causing the lowering of the limiting current, the shift of the half-wave potential, the splitting of the original wave into two waves, a minimum on the limiting current etc. Until recently these phenomena described very often in the polarographic literature have not been elucidated quantitatively. A deeper insight into the mechanism could be 
obtained by means of the so-called $i-t$ curves $^{9-12)}$ representing the dependence of the instantaneous current on the time on single drops.

The theoretical solution of the polarographic currents influenced by surface active substances was possible after some simplified assumptions have been made.

Here an analoguous model is made use as that used by Frumkin ${ }^{13)}$ for a simplified description of the properties of the capacity of an electrode which is partially covered by a surface active substance. Two kinds of electrode surface are considered here, one of which is covered by the adsorbed substance and the other is free. The electrode reaction on the covered and on the free surface proceeds at different rates. If the rate on the occupied surface is lower than that on the free one a retardation (inhibition) of the over-all electrode reaction occurs. If, on the other hand, the rate of the electrode reaction at the covered surface is higher than that at the free one, an acceleration of the over-all electrode reaction may take place. Under this assumption the rate constant of the electrode reaction in the presence of the surface active substance can be expressed as follows ${ }^{14,15}$

$$
k_{e f}={ }_{0} k_{e}(1-\Theta)+{ }_{1} k_{e} \Theta
$$

where ${ }_{0} k_{e}$ denotes the rate constant on the free, ${ }_{1} k_{e}$ that on the covered surface and $\Theta$ is the coverage expressed by the known relationship

$$
\Theta=\frac{\Gamma}{\Gamma_{m}}
$$

Here $\Gamma$ is the surface excess at a certain concentration of the surface active substance and $\Gamma_{m}$ the maximum surface excess in the case of full coverage.

The rate constant depends exponentially on the potential in the usual way. In order to obtain the corresponding expression for the current, the relation (1) must be put as a boundary condition in the system of differential equations describing the change of the concentration of the depolarizer in the neighbourhood of the electrode caused by the concentration polarization and the rate of the electrode reaction itself.

Some special cases must be now considered concerning the nature of the adsorption of the electrode reaction.

Firstly the kinetics of film formation must be taken into account. In this respect three different types of adsorption can be supposed.

a) If the adsorption is very strong and takes place rapidly, then the concentration of the dissolved surface active substance in the solution in the neighbourhood of the electrode surface is considerably diminished and it may be assumed that the surface active substance is transported to the electrode by diffusion only. The amount $N$ of the adsorbed active substance $\mathrm{A}$ in the time $t$ elapsed from the beginning of the drop may be express$\mathrm{ed}^{16)}$ by the Ilkovič equation

$$
N=\int_{0}^{t} 0 \cdot 732 m^{2 / 3} t^{1 / 6} D_{A}^{1 / 2} C_{A} d t=0 \cdot 627 m^{2 / 3} t^{7 / 6} D_{A}^{1 / 2} C_{A} .
$$

The amount adsorbed per unit area $\Gamma$ is

$$
\Gamma=\frac{N}{q}=7.36 \times 10^{-4} C_{A} D_{A}^{1 / 2} t^{1 / 2}
$$

where $C_{A}$ is expressed in mol/l and $q$ denotes the surface of the drop. The maximum amount $\Gamma_{m}$ of surface active substance in the moment $\theta$ of the complete coverage is given by the equation

$$
\Gamma_{m}=7 \cdot 36 \times 10^{-4} C_{A} D_{A}{ }^{1 / 2} \theta^{1 / 2}
$$

which can be written in the form 


$$
\hat{\theta}=1.85 \times 10^{6} \frac{T_{m}^{\prime 2}}{C_{A}{ }^{2} D_{A}} .
$$

This equation is a very simple criterion to determine, whether the adsorption process is diffusion controlled. The time $\theta$ as it will be shown later can easily be obtained from the $i$ - $t$ curves. Thus the coverage is given by a very simple expression

$$
\Theta=\frac{\Gamma}{\Gamma_{m}}=\left(\frac{t}{\theta}\right)^{1 / 2}
$$

b) In the second case the rate of the adsorption itself can be the rate determining step. A corresponding differential equation considering the rate constants of adsorption $k_{a}$ and of desorption $k_{\text {des }}$ must be taken into account. The final expression for the coverage is as follows ${ }^{17)}$

$$
\Theta=\frac{1}{1+\frac{k_{\text {des }}}{k_{a} C_{A}}} F(\tau)
$$

where

$$
\tau=\frac{k_{a} C_{A}+k_{d e s}}{\Gamma_{m}} \cdot t
$$

and $F(\tau)$ is the tabulated function.

c) In the third case the adsorbability of the surface active substance can be relatively small, so that rather high concentrations of surface active substances in the solution are required if an observable value of its surface concentration has to be attained. Thus, by the adsorption the concentration of the surface active substance at the electrode is not distinctly diminished. Then, the surface concentration is time-independent and given by the equation

$$
\frac{\Gamma}{\Gamma_{m}}=f\left(C_{A}\right)
$$

where $f\left(C_{A}\right)$ is an appropriate adsorption isotherm which may be of course a function of the electrode potential.

In all cases the adsorption of a foreign electrochemically inactive substance was considered. Now the case is being solved in which the reaction product is quantitatively adsorbed on the electrode surface during the electrochemical reaction. Hence the equation for $\Theta$ is ${ }^{18}$

$$
\Theta=\frac{A}{t^{2 / 3}-} \int_{0}^{t} t^{2 / 3} D\left(\frac{\partial C}{\partial x}\right)_{x=0} d t
$$

where $A$ is area occupied by 1 molecule adsorbed in the surface, $(\partial C / \partial x)_{x=0}$ is the concentration gradient of the depolarizer at the electrode, $D$ its diffusion coefficient.

If the corresponding relationships for $\Theta$ are inserted in the equation (1) and this equation is then put as a boundary condition in the system of differential equations for the slow electrochemical reaction, the course of the instantaneous, or the value of the average current and in some cases the relationship for a current-potential curve can be obtained.

From theoretical equations it can be shown that the influence of surface active substances on polarographic currents depends on the kinetics of the adsorption, on the charge of the surface active substance and on the degree of the reversibility of the electrode reaction of the depolarizer.

If the rate determining step of the adsorption is the diffusion of surface active substance and the electrode process is irreversible (that is $\eta_{1 / 2}=E_{1 / 2}-E^{0}>200 \mathrm{mV}$ for a one 
electron process and $100 \mathrm{mV}$ for a two-electron process), then the instantaneous current inhibited by adsorption $i_{a}$ is given by the relationship ${ }^{15)}$

$$
i_{a}=70.6 n m^{2 / 3} D^{1 / 2} C\left(1-\frac{{ }_{1} k_{e}}{{ }_{0} k_{e}}\right)^{-1 / 3} \theta^{1 / 6} H(\beta \xi, \beta) .
$$

The function $H(\beta \xi, \beta)$ is proportional to the current $i$ and the parameters $\beta \xi$ and $\beta$ are expressed as follows ${ }^{15}$ )

$$
\begin{aligned}
& \beta \xi=\frac{{ }_{0} k_{e}-{ }_{1} k_{e}}{{ }_{0} k_{e}}\left(\frac{t}{\theta}\right)^{1 / 2} \\
& \beta=\frac{{ }_{0} k_{e}-{ }_{1} k_{e}}{{ }_{0} k_{e}{ }^{2}} \sqrt{\frac{7 D}{12 \theta}} .
\end{aligned}
$$

For large values of ${ }_{0} k_{e}$ that is at the potentials of the limiting current of the original wave of the depolarizer the current should after the addition of surface active substance decrease discontinuously to value corresponding to the rate constants ${ }_{1} k_{e}$, which could be of course in some cases equal to zero, when the time $\theta$, necessary to the coverage of the electrode was reached. The experimental results are illustrated in Fig. 1. The agreement of the experimental $i$ - $t$ curves with those predicted by the theory is satisfactory.

The experimental shape of an $i-t$ curve is very close to the theoretical one, but a certain bent on the curve at the maximum and the minimum current is observed. This is due to the fact that for times near

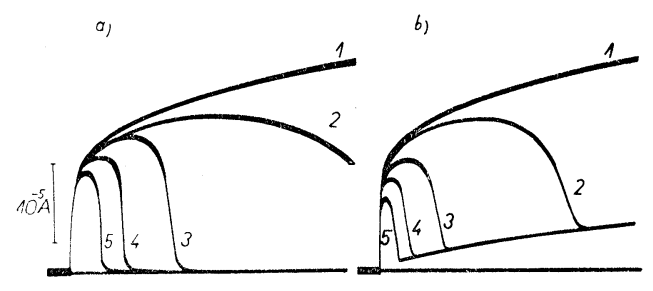

Fig. 1. I- $t$ curves in the presence of the surface active substance at the potential of the limiting current

a) ${ }_{1} k_{e}=0$; b) ${ }_{1} k_{e}>0$. a) $6 \times 10^{-3} \mathrm{MVOSO}_{4}$, $0.1 \mathrm{MH}_{2} \mathrm{SO}_{4}, E=-1.2 \mathrm{~V}$ (SCE). Concentration of tribenzylamine : 1) 0 ; 2) $5 \times$ $10^{-5} \mathrm{M}$; 3) $1 \times 10^{-4} \mathrm{M}$; 4) $1.5 \times 10^{-4} \mathrm{M}$; 5) $2 \times 10^{-4} M, t_{1}=5.4 \mathrm{sec}$. b) $7.5 \times 10^{-3} M$ $\mathrm{Ti}\left(\mathrm{SO}_{4}\right)_{2}, 0.11 M \mathrm{H}_{2} \mathrm{SO}_{4}, E=-1.2$ (SCE). Concentration of tribenzylamine : 1) 0 ;2) $8.3 \times 10^{-5} M$; 3) $1.5 \times 10^{-4} M$; 4) $2.1 \times$ $10^{-4} M$; 5) $2.6 \times 10^{-4} M ; t_{1}=5.2 \mathrm{sec}$. to $\theta$ the simplified assumptions in the theory are not fulfilled.

The most important proof of the theory is the change of the $i-t$ curves with ${ }_{0} k_{e}$ which was determined from the known relationship

$$
\frac{\bar{\imath}}{\bar{i}_{l}-\bar{\imath}}=0.886_{0} k_{e} \sqrt{\frac{t_{1}}{D}} .
$$

The change of the size and of the shape of $i$ - $t$ curves with different values of ${ }_{0} k_{e}$ is shown $^{11,19,20)}$ in Figs. 2 and 3.

If ${ }_{1} k_{e}=0$, an expression for the value of the average currents and the current voltage curves can be calculated $^{20)}$. In this case for a reduction process the diminishing of the limiting current and the shift of the polarographic curve inhibited by adsorption towards more negative potentials takes place (Fig. 4). The shift of the wave is given by the relationships

$$
\Delta E=2: 303 \frac{R T}{\alpha n F} \log \left(a \sqrt{\frac{\theta}{t_{1}}}\right) .
$$

Corresponding equations for the diminishing of the current measured at a constant potential and for the shift of the potential measured at constant average current after the addition of the surface active substance were also derived ${ }^{20)}$. In both cases the conditions were chosen, at which no concentration polarisation occurs. For the shift of the potential 


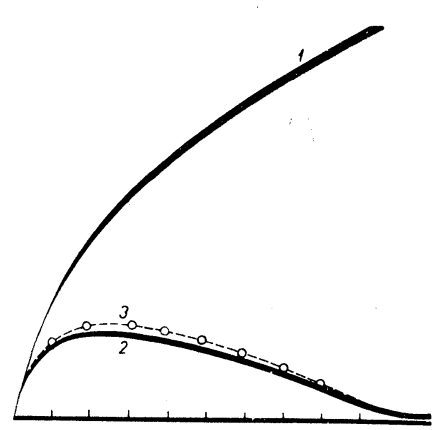

Fig. 2. I- $t$ curves of $\mathrm{Co}\left(\mathrm{NH}_{3}\right)^{3+}$ in the presence of polyvinylalcohol

1) $0.08 M \mathrm{Co}\left(\mathrm{NH}_{3}\right)_{6} \mathrm{Cl}_{3}, 0.1 M \mathrm{H}_{2} \mathrm{SO}_{4}$, $0.5 \mathrm{M} \mathrm{K}_{2} \mathrm{SO}_{4}$ for potentials corresponding to $\left.i / \bar{t}_{1}<1 / 10 ; 2\right)+0.008 \%$ polyvinylalcohol ; 3 ) theoretical curve. $t_{1}=6.2$ sec., $\theta=3.8 \mathrm{sec}$.

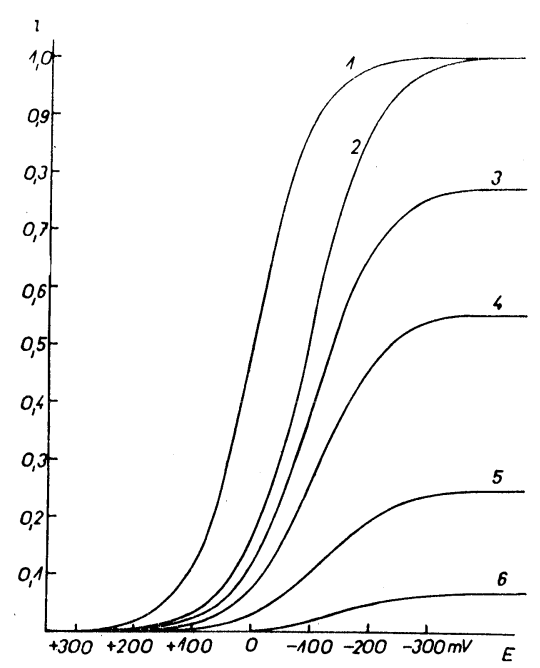

Fig. 4. The decrease of the limiting current and the shift of the wave with the coverage of the electrode

1) Irreversible wave with the slope $0,116 \mathrm{~V}$. 2) -6) calculated waves for the ratios $\theta / t_{1}$ : 2) 1 ; 3) 0.8 ; 4) 0.6 ; 5) 0.3 ; 6) 0.1 .

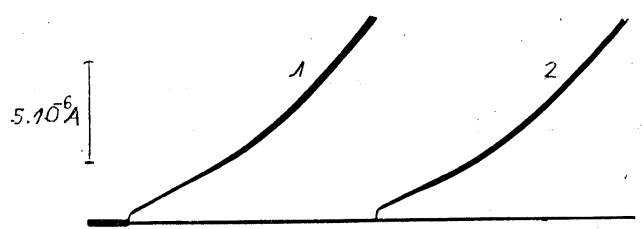

Fig. 5. I- $t$ curves showing the accelerating influence of alcaloids on the depolarization process of $\alpha$-bromopropionic acid on the foot of the polarographic wave. Phosphate buffer $\mathrm{pH} 6,9,8.3 \times 10^{-3} M \quad \alpha$-bromopropionic acid. $2 \times 10^{-5} M$ diacetylmorphine, $E=-0.95 \mathrm{~V}(\mathrm{SGE})$.

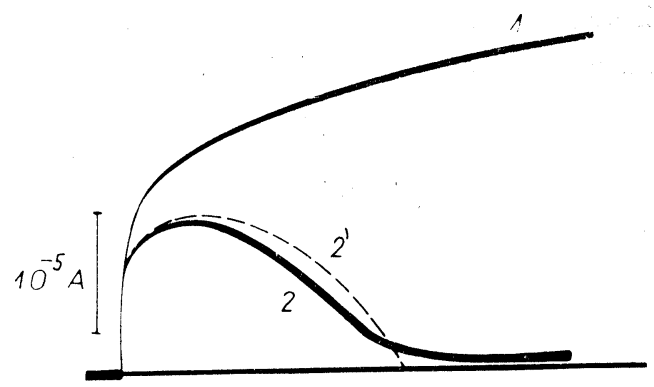

Fig. 3. I- $t$ curves of $\mathrm{Co}\left(\mathrm{NH}_{3}\right)_{6}{ }^{3+}$ in the presence of polyvinylalcohol

1) $9 \times 10^{-3} M \mathrm{Co}\left(\mathrm{NH}_{3}\right)_{6} \mathrm{Cl}_{3}, 0.1 \mathrm{M} \mathrm{H}_{2} \mathrm{SO}_{4}$, $i$ - $t$ curve at the potential of the limiting current ; 2) $+0.0055 \%$ polyvinylalcohol, $i$ - $t$ curve at the potential corresponding $\bar{\imath} / \bar{l}_{l}$ $\left.=0.9 ; 2^{\prime}\right)$ calculated $i$ - $t$ curve.

in the case of inhibition at the constant current $\bar{\imath} \ll \overline{\boldsymbol{i}}_{1}$ holds

$$
\Delta E_{1}=2.303 \frac{R T}{\alpha n F} \log \left[0.231\left(\frac{\theta}{t_{1}}\right)^{5 / 3}\right] \text {. }
$$

In all cases the agreement of our findings with the theoretical predictions was $\operatorname{good}^{20 !}$.

It must be emphasized that the derived expressions are valid only if ${ }_{1} k_{e}$ in a relatively broad potential range is practically equal to zero. Of course in many cases ${ }_{1} k_{e}>0$ and increases with the potential. Under such conditions the shape of the polarographic wave cannot be expressed by such simple equations.

If the adsorption of the surface active substance accelerates the electrode reaction a very simple equation can be derived for the case, when the concentration polarization does not occur, i.e., for the potential at the beginning of the rising portion of the polarographic wave $^{15)}$. Then the instantaneous current is given by the equation

$$
i_{a}=0.85 n F m^{2 / 3}{ }_{1} k_{e} C \theta^{-1 / 2} t^{7 / 6}
$$

and is shown ${ }^{21)}$ on the Fig. 5.

The substantial deviations of the experimental $i$ - $t$ curves from the theoretical ones were observed in the presence of surface active cations during the irreversible reduction of the cation ${ }^{11,19}$. So the $i-t$. 
curve (Fig. 6) for ${ }_{0} k_{e}$ corresponding to $i / \bar{i}_{l}=0.8$ was much less and of a shape to be expected for a smaller value of ${ }_{0} k_{e}$. This behaviour can be explained in the following way: After addition of the surface active cation the original rate constant ${ }_{0} k_{e}$ given by the expression

$$
{ }_{0} k_{e}=k \exp \left(-\frac{\alpha n F}{R T} E\right)
$$

can be written in the form

$$
{ }_{0} k_{e}^{\prime}={ }_{0} k_{e} \exp \left[\frac{-\alpha n F \Psi_{1}}{R T}\left(\frac{z}{\alpha n}-1\right)\right],
$$

where $z$ is the charge of the depolarizer. Analogically, the velocity constant ${ }_{1} k_{e}$ at the covered surface changes. We shall suppose that its value is equal to zero.

In the first approximation it is assumed that the value of $\Psi_{1}$-potential depends linearly on the coverage of the electrode

$$
\Psi_{1}=\Psi_{1}^{0}+\Delta \Psi_{1} \Theta
$$

where $\Psi_{1}^{0}$ is the value of the $\Psi_{1}$-potential at free surface, $\Psi_{1}^{0}+\Delta \Psi_{1}$ that at the complete coverage. Under this assumption the influence of the surface active substance of ionic types on the charged depolarizer was solved (Fig. 7). The derived formula is simple in a

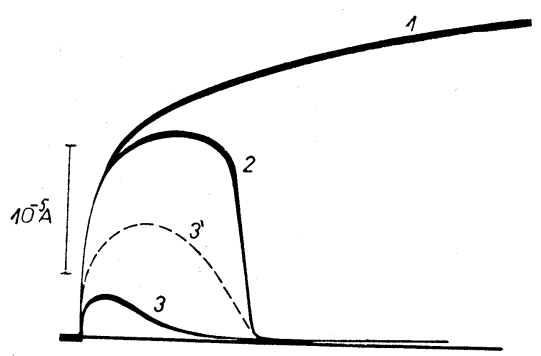

Fig. 6. I- $t$ curves of $\mathrm{VO}^{2+}$ in the presence of tribenzylamine at different potentials 1) $6 \times 10^{-3} M \mathrm{VOSO}_{4}, 0.1 M \mathrm{H}_{2} \mathrm{SO}_{4}$ at the potential of the limiting current $(E=$ $-1.34 \mathrm{~V}) ; 2)+1 \times 10^{-4} M$ tribenzylamine, at $E=-1.34 \mathrm{~V}$ (SCE) ; 3) $+1 \times$ $10^{-4} M$ tribenzylamine at the potential corresponding $\left.i / i_{d}=0.8 ; 3^{\prime}\right)$ calculated $i-t$ curve without consideration of the influence of $\Psi_{1}$-potential.

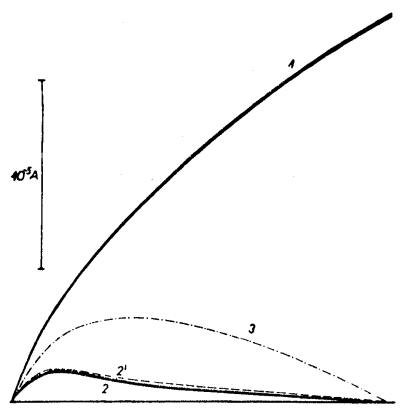

Fig. 7. I- $t$ curve of $\mathrm{VO}^{2+}$ in the presence of tribenzylamine on the foot of the polarographic curve

1) $0.06 M \mathrm{VOSO}_{4}, 0.1 M \mathrm{H}_{2} \mathrm{SO}_{4}$ :

2) $+6.8 \times 10^{-5} M$ tribenzylamine ;

$2^{\prime}$ ) theoretical $i$-t curve for $\Delta W_{1}=$ $0.04 \mathrm{~V}, z=3, \alpha_{n}=0.45, E=-0,755$ $\mathrm{V}$ (SCE) ; 3) theoretical $i-t$ curve for $\Delta \Psi_{1}=0$.

case, when no concentration polarization takes place. Then the shift of the polarization curve of the charged depolarizer due to the surface active substance of the same sign of charge, may be expressed as follows

$$
\Delta E=\Delta E_{1}+0.6\left(1-\frac{z}{\alpha n}\right) \Delta \Psi_{1}
$$

where $\Delta E_{1}$ is the shift caused by the nonpolar surface active substance with the same value of $\theta$, and the second term on the right hand side corresponds to the electrical effect.

If the surface active ion has the opposite sign to the depolarizer, the accelerating effect of the electrode reaction can take place. This problem was solved ${ }^{2}$ under the same assumptions as in the work of Kuta, Weber and Kouteck $y^{\prime 20}$ and a good agreement ${ }^{3)}$ with the theory was found.

If the electrode reaction is polarographically reversible, that is, if the rate constant 


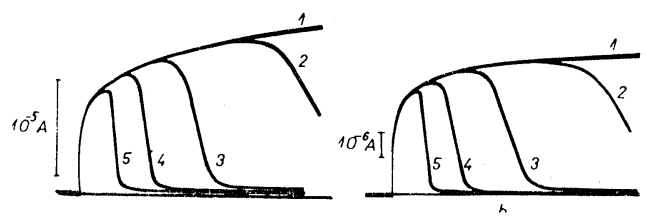

Fig. 8. I- $t$ curves of $\mathrm{Cd}^{2+}$ in $\mathrm{HClO}_{4}$ in the presence of tetrabutylammonium sulphate at different potentials

1) $2.5 \times 10^{-3} \mathrm{M} \mathrm{CdSO}_{4}, 0.1 \mathrm{M} \mathrm{HClO}_{4}$. Concentration of tetrabutylammonium sulphate : 2) $1.4 \times 10^{-5} M$; 3) $2 \times 10^{-5} M$; 4) $2.8 \times 10^{-5} \mathrm{M}$; 5) $4 \times 10^{-5} \mathrm{M}$. a) $i-t$ curves at the potential of the limiting current; b) at the potential corresponding $\bar{\imath} / \bar{\imath}_{l}=0.34$.

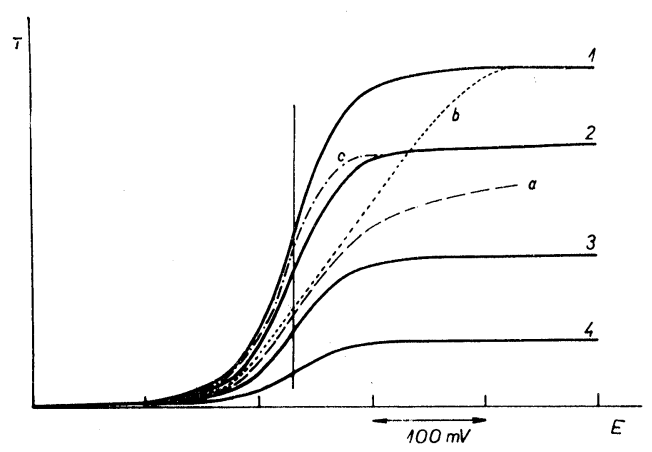

Fig. 9. The influence of surface active substance on the reversible reduction wave

1) Polarographic curve for the case of oneelectron reduction ; 2) - 4) decreased waves for different values of coverage, if ${ }_{1} k_{e} \rightarrow 0$ :

2) $t_{1} / \theta=1.25$; 3) $t_{1} / \theta=2$; 4) $t_{1} / \theta=4$. Curves a), b), c) correspond schematically to cases that ${ }_{1} k_{e}>0$; a) ${ }_{1} k_{e}$ increases slowly with the potential $\left.\left(t_{1} / \theta=2\right) ; \mathrm{b}\right){ }_{1} k_{e}$ increases considerably with the potential $\left(t_{1} / \theta=2\right)$;

c) ${ }_{1} k_{e}$ decreases with increasing potential $\left(t_{1} / \theta=1,25\right)$.

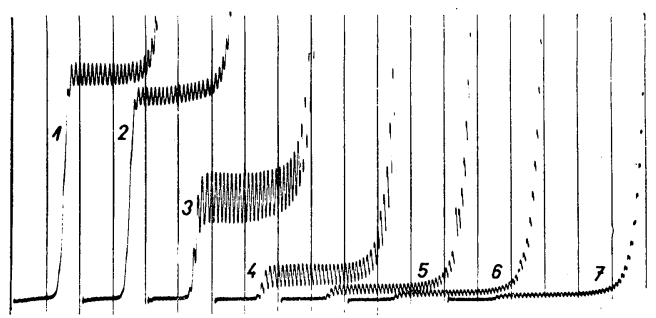

Fig. 10. Influence of tribenzylamine on the $\mathrm{Gd}^{2+}$ reduction in hydrochloric acid

1) $5 \times 10^{-4} \mathrm{M} \mathrm{CdSO}_{4}, 0.2 \mathrm{M} \mathrm{HCl}$. Concentration of tribenzylamine : 2) $5 \times 10^{-5} M ; 3$ ) $1 \times 10^{-4} \mathrm{M}$; 4) $2 \times 10^{-4} \mathrm{M}$; 5) $3 \times 10^{-4} \mathrm{M}$; 6) $4 \times 10^{-4} M$; 7) $5 \times 10^{-4} M$. Start $-0.660 \mathrm{~V}$ (vs. mercurosulphate electrode), $220 \mathrm{mV} /$ absc., sens. $1: 30$. for $E=E^{0}{ }_{0} k_{e}^{0} \geqslant 2 \times 10^{-2} \mathrm{~cm} \mathrm{sec}^{-1}$ and if ${ }_{1} k_{e} \rightarrow 0$ in a larger potential range, then the shape of $i$ - $t$ curves in the presence of the surface active substance is independent of the potential (Fig. 8), and only a decrease in the limiting current occurs without a shift of the half-wave potential (Fig. 9 and 10) and without changing the slope of the wave. If ${ }_{1} k_{e}>0$ and is relatively much increasing with the potential, then the limiting current is not diminished and so-called irreversible character of the wave is observed (Fig. 9). It was shown ${ }^{24)}$ from the $i$ - $t$ curves that after addition of surface active ion to the solution of the depolarizer with the same sign of charge, the rate constant of which attains this lower limit, the rate constant at the free surface can be diminished in such a manner that it behaves irreversibly.

As an example the discharge of $\mathrm{Cd}^{2+}$ in $\mathrm{H}_{2} \mathrm{SO}_{4}$ can be given. The rate constant of the electrode reaction as determined by different electrochemical methods is about $4 \times 10^{-2} \mathrm{~cm} \mathrm{sec}^{-1}$. Whilst in the presence of surface active substances of nonpolar character a shape of $i$ - $t$ curve was obtained corresponding to a reversible reaction, in the presence of tribenzylamine the shape of $i$ - $t$ curve shows a lowering of the rate constant on the free surface. This effect is attributed to the positive sign of the $\Psi_{1}$-potential in presence of tribenzylamine cation.

As it was already shown the influence of the adsorption consists of two effects : the first one could be denoted as a mechanical inhibition and the second one as an electrical effect.

From many results ${ }^{24}$ obtained during the study of the influence of the adsorption on electrode kinetics another example could be mentioned. As it is known the rate constant of the reduction of ferrioxalate in neutral solution of 
oxalate in the concentration $>0.2 M$ is very $\left.\operatorname{large} e^{25}\right),{ }_{0} k_{e}^{0}>1$. In this case the number of ligands of the oxidized and reduced from is the same. For smaller concentration of oxalate the decreased rate constant is attributed to the dissociation of ferrooxalate complex, nevertheless, the system behaves polarographically reversible. As it could be expected the positively charged surface active substance (e.g. tetrabutylammonium) has a small influence ${ }^{24)}$ on the reduction rate of negatively charged $\mathrm{Fe}\left(\mathrm{C}_{2} \mathrm{O}_{4}\right)_{3}{ }^{3-}$ in neutral oxalate solution. The inhibiting effect of tetrabutylammonium is considerably increased by addition of iodide, whilst iodide alone does not inhibit in this case the reduction of ferrioxalate.

The corresponding electrocapillary curves ${ }^{24}$ show the decrease of surface tension if the icdide and tetrabutylammonium are simultaneously present in the solution.

The described effects are increased if the concentration of oxalate is lowered. Under these conditions the iodide inhibits the electrode reaction of ferrioxalate.

It can be considered that in these cases the negative value of $\Psi_{1}$-potential exerts a predominant influence on the reduction of ferrioxalate complex containing 3 negative charges.

From the study of the electrical influence of the double layer on the reduction of complexes of cobalt with different ligands it could be shown ${ }^{26}$ that the whole charge of the complex is not so important as the distribution of the electrical charges in the complex. In other words the inhibiting or accelerating effect of the surface active substance depends on the orientation of the complex at the electrode.

The corresponding equations for the instantaneous current and polarization curve without concentration polarization were derived also for the case that the rate of the adsorption is the rate determining step. The maximum on the $i-t$ curve should be indirectly proportional to the concentration of the surface active substance. Experimental verification for this case and for the case when the adsorption of reduced form influences an irreversible reaction is not described with certainty.

Finally, examples of the influence of the adsorption on the substitution reactions will be given. The rate constant of the chemical substitution reaction of the type

$$
\mathrm{Co}\left(\mathrm{NH}_{3}\right)_{5} \mathrm{X}^{2+}+\mathrm{Cl}^{-} \stackrel{k}{\longrightarrow} \mathrm{Co}\left(\mathrm{NH}_{3}\right)_{5} \mathrm{Cl}^{2+}+\mathrm{X}^{-}
$$

measured chemically was found to be relatively slow, of the order $10^{-5}(\mathrm{~mol} / 1)^{-1} \mathrm{sec}^{-1}$. When studying this reaction polarographically ${ }^{27}$, an extremely large value of this constant determined from the current corresponding to the reduction of $\left[\mathrm{Co}\left(\mathrm{NH}_{3}\right)_{5} \mathrm{Cll}\right]^{2+}$ was found and this value increases in the series $\mathrm{Cl}^{-}, \mathrm{Br}^{-}, \mathrm{I}^{-}$. An assumption was made, namely, that the reaction proceeds with a quite different rate, being catalysed by the adsorption at the surface of the electrode. Such catalysis of reactions of complexes has been observed already in some cases, the catalyst being charcoal, silica gel, mercury, Raney nickel etc.

As a further example ${ }^{28}$ the reaction between Co(II)hexamo-complexes and cystein could serve. From the study of $i$ - $t$ curves and temperature dependences, it can be concluded that the adsorption plays here the predominant role.

It must be emphasized that the present treatment representing the first quantitative approach to the complicated problem of the influence of adsorption in polarography is based on rather simplified assumptions. Nevertheless, the agreement of experimental results with theoretical ones was in many cases satisfactory. It can be supposed that the 
further work on the properties of the double layer which is now in progress in many laboratories will contribute to the elucidation of adsorption phenomena in polarography.

\section{References}

1) P. Delahay, Nere Instrumental Methods in Electrochemistry, Interscience, New York, 1954.

2) K.J. Vetter, Elektrochemische Kinetik, Springer, Berlin, 1961.

3) N.V. Nikolayeva-Federovich, N.V. Fokina and O.A. Petrii, Doklady Akad. Nauk SSSR, 122, 639 (1958).

4) A.N. Frumkin, Trans. Fadaday Soc. 55, 156 (1959).

5) A.N. Frumkin, B.B. Damaskin and N.V. Nikolayeva-Fedorovich, Doklady Akad. Nauk SSSR 115. 751 (1957).

6) B.B. Damaskin, N.V. Nikolayeva-Fedorovich and A.N. Frumkin, ibid. 121, 129 (1958).

7) G.N. Reilley and W. Stumm, Progress in Polarography, Volume I, p. 81, Interscience, New York, 1962.

8) H.W. Nürnberg, and M.v. Stackelberg J. Electroanal. Chem. 4, 1 (1962).

9) I. Smoler and J. Ku ta, Z. physik. Chem. (Leipzig), Sonderheft 1958, 58.

10) J. K ůta and I. Smoler, Collection Czechoslov. Chem. Commun. 24, 2208 (1959).

11) J. K ůta and I. Smoler, Advances in Polarography, p. 350, Pergamon Press, London, 1960.

12) J. Küta and I. Smoler, Progress in Polarography, Volume I, p. 43, Interscience, New York, 1962.

13) A.N. Frumkin, Z. Physik 35, 792 (1926).

14) R.W. Schmid and G.N. Reilley, J. Am. Chem. Soc. 80, 2087 (1957).

15) J. Weber, J. Koutecký and J. Koryta, Z. Elektrochem. 63, 583 (1959).

16) J. Koryta, Collection Czechoslov. Chem. Commun. 18, 206 (1953).

17) J. Weber and J. Koutecký, ibid. 25, 2993 (1960).

18) B. Tvaroha, in course of publication.

19) J. Kůta and I. Smoler, Z. Elektrochem. 64, 285 (1960).

20) J. K ůta, J. Weber and J. Koutecký, Collection Czechoslov. Chem. Commun. 25, 2376 (1960).

21) V. Volková, Nature 185, 743 (1960) ; Advances in Polarography, p. 840, Pergamon Press, London 1960.

22) A. Ya. Gokhshtein, Doklady Akad. Nauk SSSR 137, 345 (1961).

23) A.N. Frumkin, O.A. Petrii and N.V. Nikolayeva-Fedorovich, ibid. 136, 1158 (1961).

24) J. K ůta and I. Smoler, Collection Czechoslov. Chem. Commun. 27, 2349 (1962).

25) J.E.B. Randles and K.W. Somerton, Trans. Faraday Soc. 48, 937 (1952).

26) J. K ůta, unpublished results.

27) A.A. Vlček and J. K ůta, Nature 185, 95 (1960).

29) M. Zielinski and J. $\mathrm{K}$ u ta, in course of publication. 